\title{
Photoacoustic for Oral Soft Tissue Imaging based on Intensity Modulated Continuous-Wave Diode Laser
}

\author{
Rini Widyaningrum ${ }^{\#}$, Dewi Agustina ${ }^{* 1}$, Munakhir Mudjosemedi ${ }^{* 2}$, Mitrayana ${ }^{* 3}$ \\ ${ }^{\#}$ Doctoral Program, Faculty of Dentistry, Universitas Gadjah Mada, \\ Jl. Denta, Sekip Utara, Yogyakarta, Indonesia, \\ E-mail: rinihapsara@ugm.ac.id \\ $*^{l}$ Department of Oral Medicine, Faculty of Dentistry, Universitas Gadjah Mada, \\ Jl. Denta, Sekip Utara, Yogyakarta, Indonesia \\ E-mail:dewi_agustinafkg@ugm.ac.id \\ *2Department of Dentomaxillofacial Radiology, Faculty of Dentistry, Universitas Gadjah Mada, \\ Jl. Denta, Sekip Utara, Yogyakarta, Indonesia \\ E-mail: munakhirms@yahoo.com \\ . *3Department of Physics, Faculty of Mathematics and Natural Sciences, \\ Universitas Gadjah Mada, Sekip Utara, Bulaksumur, Yogyakarta, Indonesia \\ E-mail: mitrayana@ugm.ac.id
}

\begin{abstract}
We built the photoacoustic imaging (PAI) systems to image oral soft tissue. To reduce the cost and size significantly, the PAI system used an intensity-modulated continuous-wave $(\mathrm{CW})$ diode laser with a wavelength of 532nm and output peak power of $200 \mathrm{~mW}$ as an excitation source, combined with a condenser microphone as photoacoustic signals detector. The Pulse Width Modulation technique was applied to form a square wave fluctuation of laser radiation by using certain duty cycle on singlefrequency of $17.8 \mathrm{kHz}$. Sample of this study was Sprague Dawley rats tongue on plasticine media, irradiated by modulated CW diode laser. The result of this study showed that modulated laser exposure on one certain spot of the sample produced PA-signals in polynomial correlation with a duty cycle of laser modulation. Based on it, the photoacoustic imaging then was done by using gradual duty cycles, i.e., $20 \%, 30 \%$, and $40 \%$. This study also showed that using $\mathrm{CW}$ diode laser which is modulated with low duty cycle can produce most accurate PA image, as well as keeping the sample from the high energy of laser exposure that may cause biological changes. Furthermore, the maximum duty cycle to modulate laser for oral soft tissue imaging in this system was $30 \%$.
\end{abstract}

Keywords - photoacoustic imaging; diode laser; oral; soft tissue; modulated laser

\section{INTRODUCTION}

The technology of medical imaging is advancing on tremendous growth during the last century. Today, imaging modalities are not only limited to X-ray plain radiography. Other imaging modalities, i.e., ultrasonography (USG), computed tomography (CT), magnetic resonance imaging (MRI), positron emission tomography (PET), and singlephoton emission computed tomography (SPECT) also have been well known by dentist and physician. Each modality has its advantages and disadvantages [1]. X-ray imaging, CT, and PET use ionizing radiation, which can cause detrimental side effects in the human body [2]-[3]. Magnetic resonance imaging utilizes strong magnetic field to image patient's condition so that every medical tool on MRI room should make of diamagnetic materials.

Dentists and physicians use imaging modalities to improve patient care as well as to reveal information about patient's condition that cannot be seen by naked eye in clinical examination. X-ray imaging modalities are the most widely used by dentists to examine teeth, jaw, and maxillofacial structures [4], but these modalities cannot serve adequate information about oral and maxillofacial soft tissue. To overcome the limitation, previous studies had also developed digital image processing such as image enhancement [5] and edge detection [6] for X-ray images to 
assist dental and medical practitioners in identifying disease and abnormalities in hard tissue and soft tissue.

In developed countries, oral radiologists use USG, MRI, and PET to image oral and maxillofacial soft tissue. Nevertheless, in Indonesia and other developing countries, these imaging modalities are high cost and limited only in individual hospitals. Beside those conventional imaging modalities, recent studies have shown new emerging biomedical imaging techniques [3],[7] referred as Photoacoustic Imaging (PAI) that potential to image soft tissue. Previous studies [3],[8]-[9] have utilized PAI technique to image normal and pathological condition in oral and other soft tissue.

Photoacoustic imaging, also known as optoacoustic imaging [10]-[11] or thermoacoustic imaging [12], is an imaging technique based on the principles of the photoacoustic (PA) effect. The PA effect refers to the conversion of light to sound [10], a phenomenon of generating acoustic flourishes from an object being illuminated by pulsed or intensity-modulated continuouswave (CW) electromagnetic waves, especially light [13]. The PA effect was first explored by Alexander Graham Bell in 1880 [10],[14]-[15], while PAI technique was first introduced as a biomedical imaging modality in 1981 by Theodore Bowen [15]-[16]. Rapid development in both theoretical and technological PAI began in the 1990s [17][18], triggered by the development of the laser, computer, and ultrasound transducer technologies in last two decades [17].

Photoacoustic imaging is a hybrid technology that integrating optical imaging and ultrasound imaging [19]-[20], links rich optical contrast and high ultrasonic resolution in a single modality [16]. This imaging technique exposes the tissue to non-ionizing radiation (e.g., lasers, radio waves, or microwaves) and measures optically induced acoustic (or ultrasound) signals [10]. Photoacoustic imaging is promising to overcome some drawbacks of other mainstream biomedical imaging modalities. PAI has rich optical contrasts and free of speckle artifacts. Different from X-ray CT and PET, PAI uses non-ionizing laser illumination. Similar with MRI and ultrasound imaging, PAI can image soft tissue as well, but PAI is faster and less expensive than MRI [21].

A typical PAI system consists of 2 main components: a laser and a microphone or a piezoelectric detector [14],[22]. There are two ways to generate photoacoustic signals: by using modulated or pulsed laser as excitation sources [22]. Tuneable nanosecond pulsed lasers such as Q-switched Nd: YAG pumped, an optical parametric oscillator (OPO), Ti: Sapphire, or dye laser are most commonly used as excitation sources in widefield photoacoustic imaging [23, 24]. Those lasers are relatively complex, expensive [25], and bulky in size [24],[26], which limits the applications of PAI technologies [19] in the field of medicine, dentistry, and biomedical. Meanwhile, diode lasers can address some of these drawbacks as they are low cost, highly compact, power efficient [23], and could be used as an active excitation source for PAI [19],[24],[26].

We developed a prototype of PAI system using diode laser as an excitation source and a condenser microphone as PA signal detector. This study investigated the usability of intensity-modulated continuous-wave $(\mathrm{CW})$ diode laser in PAI system to image oral soft tissue.

To develop the system, before the study we investigated the correlation between laser intensity-modulation and PA signal. The laser light intensities were determined by its duty cycle. In consideration of data resulting by the prior study, we determined the range of laser intensity-modulation to image the sample and investigated the influence of laser light intensity-modulation to the photoacoustic image (PAimage) quality. Furthermore, this study was done to get the most appropriate intensity-modulation of diode laser excitation in the system to produce optimum PA-image without any biological changes on oral soft tissues.

\section{MATERIAL AND METHOD}

\section{A. The PAI System}

This study was conducted at Faculty of Mathematics and Natural Sciences, Universitas Gadjah Mada. The study protocols were approved by the Medical and Health Research Ethics Committee, Faculty of Medicine, Universitas Gadjah Mada-Dr Sardjito General Hospital, Yogyakarta, Indonesia (Ref: KE/FK/036/EC/2016).

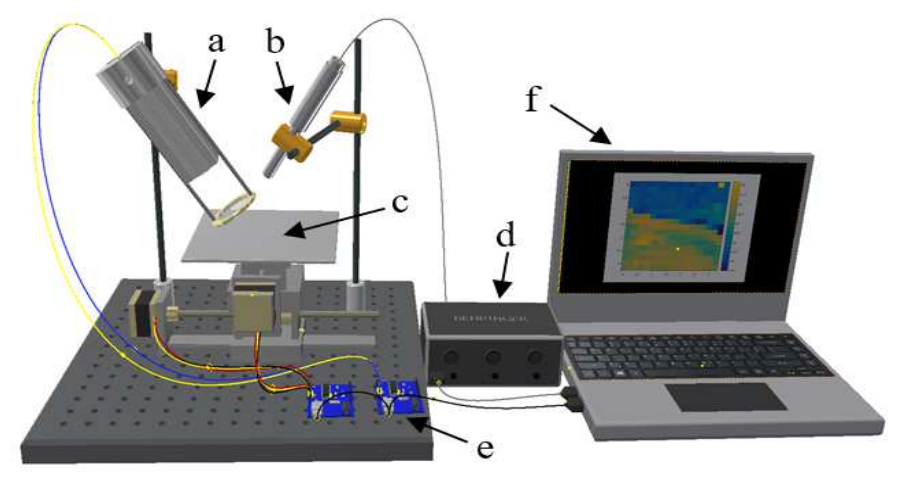

Fig. 1 The prototype of PAI system for oral soft tissue imaging. The system used intensity modulated CW diode laser as PA excitation source (a), condenser microphone as detector (b), a custom-built X-Y stage (c), an USB audio interface with mic preamplifier (d), microcontroller (e), and a portable computer (f) for signal data acquisition, data processing, and PAimage reconstruction.

The PAI system configuration is shown in Fig.1. A diode laser with a wavelength of $532 \mathrm{~nm}$ and output peak power of $200 \mathrm{~mW}$ was used as an excitation source. The laser beam was focused onto the imaged sample by using a convex lens (focal length $100 \mathrm{~mm}$ ). The distance between the lens and the imaged sample adjusted to achieve the optimal focused laser beam. The diode laser was controlled by a TTL input connected with a microcontroller to produce modulated laser excitation. Laser modulation in this study applied the Pulse Width Modulation (PWM) technique to form a square wave fluctuation in the laser TTL input so that the laser turned on and off periodically. The fluctuation of laser radiation was produced by setting certain duty cycle on a single-frequency of $17.8 \mathrm{kHz}$. The higher duty cycle applied in modulation, the higher intensity of the laser exposure.

A measurement condenser microphone ECM8000 (Behringer, Germany) was used as a detector to record the PA-signal generated from the sample. The detector was connected to a portable computer by using a USB audio 
interface with mic preamplifier U-Phoria UMC 202HD (Behringer, Germany).

Two-dimensional (2D) scanning of the sample was performed with a computer-controlled custom built X-Y stage that moved the sample in $\mathrm{X}$ and $\mathrm{Y}$ directions. PAsignal data acquisition, data processing, and PA-image reconstruction were performed in a portable computer. The PAI system recorded the PA signals at each point of the ROI in the sample and performed 2D-scanning in the horizontal $\mathrm{X}-\mathrm{Y}$ plane with a step size of $200 \mu \mathrm{m}$ between points.

B. A sample of the Study

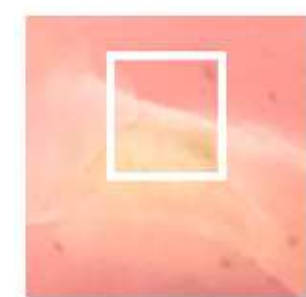

A

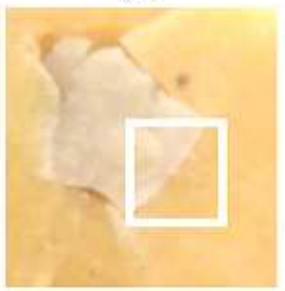

C

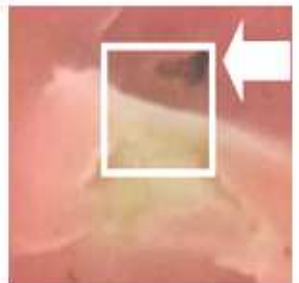

B

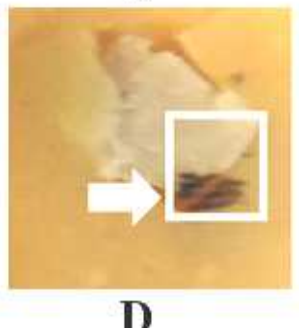

D
Fig. 2 Sample 1 (A and B) and sample 2 (C and D). Samples of the study were oral soft tissue in plasticine, before (A and C) and after (B and D) imaged by the PAI system. The ROI was marked with white-square. There was a burnt area (marked with a white arrow) on ROI after illuminated with high $(30-40 \%)$ duty cycle of laser modulation.

The sample in this study was oral soft tissue placed in plasticine (Fig. 2). The plasticine was used to place the oral soft tissue to get a flat surface and to ensure the distance of sample-detector and sample-laser was evenly constant in the imaged area. Oral soft tissues which were used for the sample in this study were Sprague-Dawley rats tongue, sliced on the sagittal plane.

\section{The Prior Study: One-Point Laser Irradiation}

This prior study was done to investigate the correlation between graduate variations of laser modulation on PAsignals. In this study, the laser beam was focused onto one certain point on oral soft tissue sample using graduate laser modulation, from the highest $(90 \%)$ to the lowest $(10 \%)$ duty cycle. The duration of laser exposure and PA-signal recording was 1 second simultaneously for each grade of intensity-modulation.

There were three samples used in the prior study. The PAsignal generated from each sample which was illuminated by using modulated $\mathrm{CW}$ laser was recorded five times for each grade of laser modulation. After that, the mean value of PAsignals from each grade of laser modulation was plotted in the graphic (Fig. 3). Data from this prior study were used to determine the optimal range of duty cycle applied in CW diode laser modulation to image samples in this study, i.e., $20 \%, 30 \%$, and $40 \%$.

\section{Photoacoustic Imaging}

To image the sample, it was placed on the X-Y stage. To generate PA-signal and form the PA-image, PAI scanning was done in the Region of Interest (ROI) of the sample. The ROI was $11.56 \mathrm{~mm}^{2}$ width and marked with white-square in Fig. 2. To ensure that our system was able to differ oral soft tissues and plasticine media surround them, the ROI covering both of oral soft tissue and plasticine in the sample. For each sample, PA-imaging was done three times by using regular duty cycles to modulated laser intensity, i.e., $20 \%$, $30 \%$, and $40 \%$ that were determined based on one-point laser irradiation experiment in the prior study.

The acquired PA-signals in PA-imaging were transferred from the detector to a computer via a USB audio interface with mic preamplifier that connected with one of the USB ports. The PA-signal from a single point in the ROI was saved in the computer as the 1-second duration of audio-data.

The software in the PAI system applied the Fast Fourier Transform (FFT) (Eq. 1) to process audio-data and produce a sound spectrum of PA-signal by transforming data in the form of time function into frequency function.

$$
X_{k}=\sum_{n=0}^{N-1} x_{n} \cdot e^{-i 2 \pi k n / N}
$$

Which $X_{k}$ is frequency function as the result of the transform, $x_{n}$ is time function, $N$ is sampling rate, and $k$ is frequency.

The PA-image was generated from PA-signal intensity peak that produced from the Fast Fourier Transformation. The peak intensity of PA-signal around the frequency of $17.8 \mathrm{kHz}$ then was taken as PA-signal value as well as an element of a matrix. By converting the matrix into a graphic, the system then reconstructed a two-dimensional PA-image based on the PA signal intensity of each point in the ROI.

\section{RESULT AND DISCUSSION}

\section{A. Result}

\section{1) PA-signal of the one point laser irradiation}

The result of the previous experiment in this study is shown in Fig. 3. Figure 3 shows that intensity-modulation of $\mathrm{CW}$ diode laser excitation in this study has a polynomial correlation with PA-signals generated from 1 particular point of the ROI.

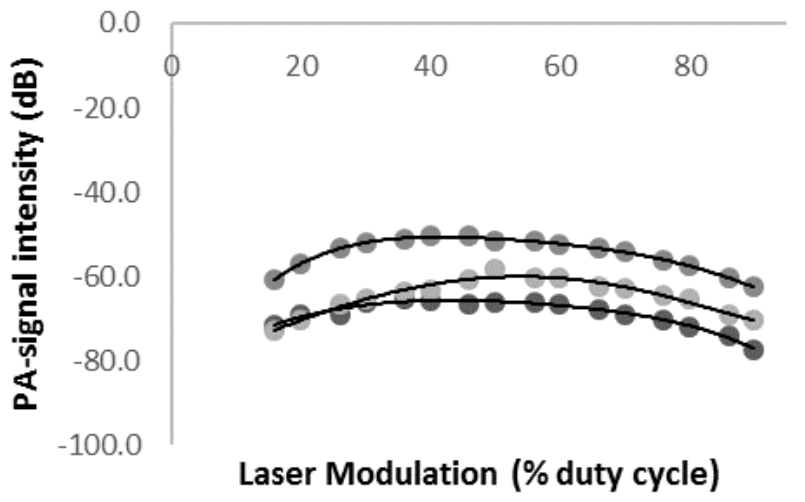

Fig. 3 PA signal from one point of oral soft tissue on 532nm CW diode laser excitation modulated with various duty cycle. 
The PA-signals tend to increase in laser excitation of 10$40 \%$ duty cycle modulation. However, modulated laser illumination using more than $40 \%$ duty cycle tend to produce constant or lower PA-signals intensities than lower duty cycles. There was no significant biological effect of laser light irradiation observed on the samples in this prior study.

2) Influence of various gradual intensity-modulated $C W$ diode laser on PA-image

Based on the result of the prior study (Fig. 3), we determined the range of duty cycle for $532 \mathrm{~nm}$ diode laser modulation with output peak power of $200 \mathrm{~mW}$ use to image the sample in this study were around $20-40 \%$. To investigate the influence of laser intensity-modulation on PAI image, the PAI system was used to produce images of samples by employing various graduate duty cycles.

Images produced by the PAI system in this study are shown in Fig. 4 and Fig. 5. The PA-images were produced by using graduate duty cycles of laser modulation (20\%, $30 \%$, and $40 \%$ ). The PA-image reconstruction was based on PA-signal intensities from every point in the ROI.

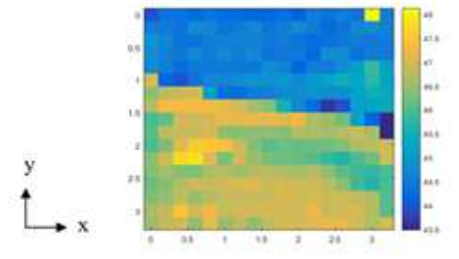

$\mathbf{A}$

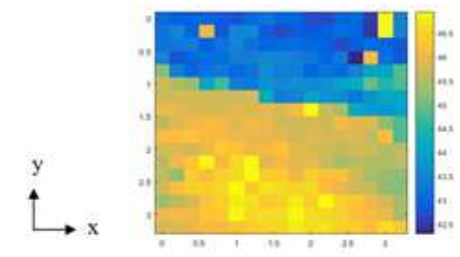

B

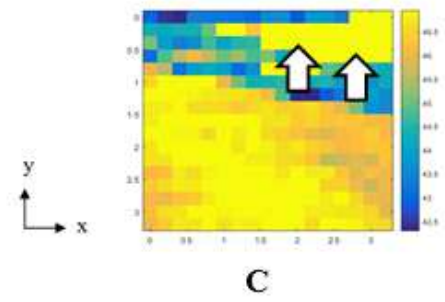

Fig. 4 PAI images from the sample (Fig. 2A), using 20\% (A), 30\% (B), and $40 \%$ (C) duty cycle intensity modulation of $532 \mathrm{~nm} \mathrm{CW}$ diode laser.

High PA-signal intensities showed brighter color than lower PA-signal intensities. The color of the PA-image represented the PA-signal intensities of every point in the ROI, as shown in the color bar on the right side of the PAimage. It was marked with dark blue for the lowest PAsignal intensity and gradually turn into light yellow for the highest intensity. Figure 4 and Fig. 5 shows that oral soft tissue produces higher PA-signals than plasticine in the same ROI, as well as brighter color in PA-images.

Focusable diode laser illumination in this study may produce a side effect, i.e., the burnt effect in the sample. The burnt effect may found in both of oral soft tissue and plasticine media while laser exposure is done in high duty cycles of modulation, i.e., $30-40 \%$. The burnt area in the
ROI produced the highest PA-signal as well as brightest yellow colour on PA-images (white arrow in Fig. 4 and Fig. 5). Moreover, PA-images of burnt samples were not able to depict the real condition of the ROI (Fig. 4B, 4C, 5B, and 5C). Photoacoustic signals from the burnt area on samples produced very high intensities compare with surrounding area in the ROI. This phenomenon decreased PA-image quality because the color bar setting become less accurate and PA-images may not show the differences between biological-tissue with the surround non-biological material.

Based on the result of the study (Fig. 4A and Fig. 5A), lowest duty cycle produced most accurate PA-image than higher duty cycles. Figure 4A and Fig. 5A show PA-images that resemble the real imaged ROI on the sample. The PAimage produced from higher duty cycles may not depict the real condition of the ROI, so that the oral soft tissue cannot well differ from plasticine in the same PA-image.

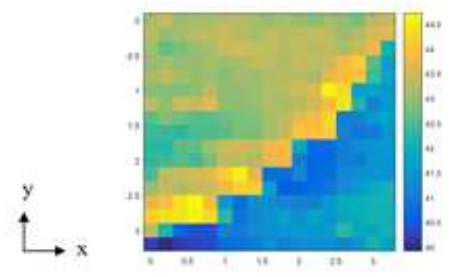

$\mathbf{A}$

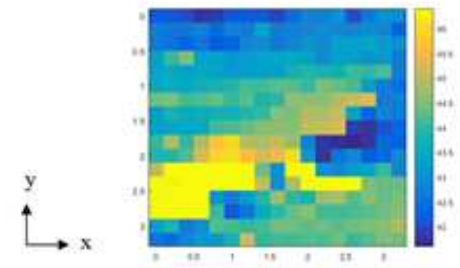

B

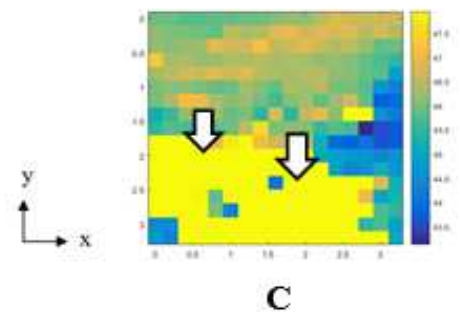

Fig. 5 PAI images from sample 2 (Fig. 2C), using 20\% (A), 30\% (B), and $40 \%$ (C) duty cycle intensity modulation of $532 \mathrm{~nm} \mathrm{CW}$ diode laser. The burnt area showed highest PA-signal on PAI images (pointed with white arrow).

\section{B. Discussion}

This study demonstrated the use of intensity-modulated CW diode laser in PAI system for oral soft tissue imaging. Two-dimensional (2D) images of oral soft tissue on sagittal section located in plasticine were obtained. This study also discovered the potential harm of the laser light to the oral soft tissue i.e. burnt effect, especially in the exposure of focusable intensity modulated $\mathrm{CW}$ diode laser with a wavelength of $532 \mathrm{~nm}$ that was used in the PAI system.

The biological tissue has optical (or photo-) absorber molecules called endogenous chromophores such as hemoglobin, melanin, water, and lipids [27]. As photons of light travel in tissue, some of them are absorbed by chromophores, and their energy is wholly or partially converted rapidly into heat [20]. The heat then produces a 
small temperature and pressure increase (about less than 0.1 $\mathrm{K})$-well below that necessary to trigger physical damage or physiological changes to tissue. This thermoelastic expansion [12] leads to an initial pressure increase, which subsequently relaxes resulting in the emission of acoustic waves. The later propagate to the surface where they are detected either by an ultrasonic transducer or a microphone, to form a PA-image which maps the original optical energy deposition within the acoustically imaged region of the sample [12],[20],[27].

In this study, PA-images showed that biological tissue especially oral soft tissue produce different PA-signal from the non-biological material, i.e. plasticine. Furthermore, the oral soft tissue can be easily differed from plasticine in PAimage by discerning the color of PA-image which represent its PA-signal intensity.

Photoacoustic imaging is more frequently use pulsed lasers excitation because they produce higher PA signal-tonoise ratio (SNR) than modulated CW excitation [20]. Unlike single-frequency $\mathrm{CW}$ excitation, pulsed excitation can provide axial resolution. The acoustic flight time in pulsed excitation provides depth information about the absorbing targets. The axial resolution of pulsed excitation is jointly determined by the laser pulse width, frequencydependent acoustic attenuation of the tissue, and a detection bandwidth of the ultrasonic transducer [20]. Even though, the pulsed photoacoustic technique requires relatively complicated and more expensive lasers, suffers from laser jitter noise and has a limited dynamic range of broadband detection [25].

Despite its advantages of compact size, low cost, and high efficiency, one drawback of diode laser as the excitation source in $\mathrm{CW}$ photoacoustic technique is limited intensities of light allowed to be used in this method for medical purposes [25]. Higher duty cycles of laser modulation in this study produced higher intensities of laser light to be absorbed by the chromophores in the sample. The superficial tissue dosage in laser irradiation is expressed influence, or energy density, measured in joules per square centimeter $(\mathrm{J} / \mathrm{cm} 2)$. Multiplying the output power of the laser in milliwatts by the time of exposure in seconds equals the produced energy. The fluence is in an inverse relationship with the laser spot size. The broader size of the irradiated area, the lower of fluence produced by the laser beam [28]. It can be assumed that for the same laser power, irradiation of focusable laser produces higher fluence than a wide laser beam. Despite producing lower fluence, pulse photoacoustic technique usually utilizes more complicated signal acquisition systems that employ high-cost detector.

Although laser light is not a form of ionizing radiation, high-intensity laser irradiation on smaller spot size may produce biological changes in the sample as found in this study. Therefore focusable laser is commonly used in dental and medical surgery [28]. Otherwise, this study utilized the focusable $\mathrm{CW}$ diode laser with low-intensity modulation to build the PAI system. Further study is suggested to consider to use of lower laser power to reduce the risk of dangerous effect in the soft tissue.

In agreement with a previous study [25], this study showed that using high-intensity diode laser which is modulated with low duty cycle can produce relatively stronger PA signals while keeping the biological sample from exposure to high energies of laser light. The PA-image (Fig. 4 and Fig. 5) resulting from this study show that low duty cycle produced more reliable image than the higher one. The later may produce a burnt effect that consequently affects the PA-image. The burnt area was imaged as an artefact in the PA-image, reconstructing from very high PAsignal intensities, far above from the mean intensities of the imaged area. This phenomenon affects the image reconstruction so that the PA-image of the burnt sample cannot resemble the real condition of the ROI.

To make a modulated laser excitation, laser radiation is employed whose intensity fluctuates periodically in the form of a square or a sine wave, resulting in a particular duty cycle [22]. Laser modulation in this study formed a square wave fluctuation by using microcontroller connected with TTL pin in laser driver. The modulation frequency was 17.8 $\mathrm{kHz}$, generated sound waves in the audible range, which can be detected by a condenser microphone. This technique is the most common sources for photoacoustic gas phase analysis [22], but this study showed that it might also be applied to solid material imaging.

Pulsed or modulated laser irradiation can generate the photoacoustic effect onto or inside a liquid, gaseous, and solid sample. Subsequently, the detection may also take place inside or at an interface of the sample. The heat inside a solid or liquid sample induced by laser irradiation then propagate to the interface so that the (photo)-acoustic detection takes place in the gas phase adjacent to the sample. This phenomenon is so-called the indirect photoacoustic generation [22].

The previous study showed that single frequency sources are applied in raster scan mode to localizing the underlying photo-absorbers. These methods enable spatial tracking in conjunction with acoustic focusing approaches, due to the phase ambiguity problem [29]. Instead of reconstructing an image digitally which commonly used in photoacoustic computed tomography (PACT) technique, a focused condenser microphone was used in this study for analog image reconstruction. This technique is most commonly used in photoacoustic microscopy (PAM) [20]. There was no digital image processing applied in this study, the PA-image (Fig. 4 and Fig. 5) was generated by using analog reconstruction. Furthermore, this aspect is recommended to be considered in further study to improve the PA-image quality.

The other drawback of $\mathrm{CW}$ photoacoustic technique is the detection in this method is more sensitive to the thermal and other environmental noises compared to pulsed PA techniques [25]. Although $\mathrm{CW}$ lasers are less expensive, single-frequency $\mathrm{CW}$ excitation cannot separate signal contributions from absorbers at different depths, unless chirped modulation or other wide-band techniques are used [20].

Many pioneering studies on PAI are based on homefabricated signal acquisition systems that employ ultrasound transducer [12]. Researchers have recently made efforts on accelerating the imaging speed of PAI drastically by developing custom designed PA-signal detectors. Most of these detectors, however, are very costly and also difficult to be duplicated by other groups when fabrication of 
sophisticated data acquisition circuits is involved [12]. Instead of using an ultrasound transducer, this study used simpler and lower cost detector that is a condenser microphone to detect audible acoustic PA-signals generated from the sample. By using a condenser microphone, the PAI system in this study can produce the PA-image that depict the oral soft tissue clearly from the non-biological material. However, to obtain higher quality of PA-images, developing the PA-detector for the PAI system become exciting challenge for the next study.

\section{CONCLUSIONS}

The PAI system developed in this study demonstrated the feasibility of modulated $\mathrm{CW}$-diode laser combined with a condenser microphone to image oral soft tissue. Based on the result of this study, we suggest $20 \%$ up to maximum $30 \%$ duty cycle to modulate the $532 \mathrm{~nm}$ continuous-wave diode laser with output peak power of $200 \mathrm{~mW}$ to image oral soft tissue. Further study need to be held to get the best intensity-modulation for oral soft tissue imaging using continuous-wave diode laser. The PAI-system in this study is also continuously developed to image oral pathological conditions, instead of normal oral soft tissue sample as imaged in this study.

\section{ACKNOWLEDGMENT}

We would like to thank The Indonesian Ministry of Research, Technology, and High Education and also to The Faculty of Dentistry, Universitas Gadjah Mada who have supported part of the funding for this study. We acknowledge Harry Miyosi Silalahi for his assistance with Fig. 1.

\section{REFERENCES}

[1] K. S. Valluru, B. K. Chinni, and N. A. Rao, "Photoacoustic Imaging: Opening New Frontiers in Medical Imaging," J. Clin. Imaging Sci., vol. 1, no. 24, pp. 1-7, 2011.

[2] R. R. Shantiningsih and S. F. Diba, "Efek Aplikasi Patch Gingiva Mukoadesif $\beta$-Carotene Akibat Paparan Radiografi Panoramik," Maj Ked Gi Ind, vol. 1, no. 2, pp. 186-192, 2015.

[3] J. Laufer, P. Johnson, E. Zhang, B. Treeby, B. Cox, B. Pedley, and P. Beard, "In vivo preclinical photoacoustic imaging of tumor vasculature development and therapy," J. Biomed. Opt., vol. 17, no. 5, p. 056016.1-8, 2012.

[4] M. Mudjosemedi, R. Widyaningrum, and R. S. Gracea, "Perbedaan Hasil Pengukuran Horizontal pada Tulang Mandibula dengan Radiograf Panoramik," Maj Ked Gi Ind, vol. 1, no. 1, pp. 78-85, 2015.

[5] J. Na'am, J. Harlan, S. Madenda, and E. P. Wibowo, "The Algorithm of Image Edge Detection on Panoramic Dental X-Ray Using Multiple Morphological Gradient (mMG) Method," Int. J. Adv. Sci. Eng. Inf. Technol., vol. 6, no. 6, pp. 1012-1018, 2016.

[6] J. Santony and J. Na' am, "Infiltrate Object Extraction in X-ray Image by using Math-Morphology Method and Feature Region Analysis," Int. J. Adv. Sci. Eng. Inf. Technol., vol. 6, no. 2, pp. 239-244, 2016.

[7] D. Wu, L. Huang, M. S. Jiang, and H. Jiang, "Contrast Agents for Photoacoustic and Thermoacoustic Imaging: A Review," Int. J. Mol. Sci., vol. 15, pp. 23616-23639, 2014.
[8] H. Fatakdawala, S. Poti, F. Zhou, Y. Sun, J. Bec, J. Liu, D. R. Yankelevich, S. P. Tinling, R. F. Gandour-Edwards, D. G. Farwell, and L. Marcu, "Multimodal in vivo imaging of oral cancer using fluorescence lifetime, photoacoustic and ultrasound techniques," Biomed. Opt. Express, vol. 4, no. 9, pp. 1724-1741, 2013.

[9] Y. Lao, D. Xing, S. Yang, and L. Xiang, "Noninvasive photoacoustic imaging of the developing vasculature during early tumor growth," Phys.Med. Biol., vol. 53, pp. 4203-4212, 2008.

[10] K. S. Valluru and J. K. Willmann, "Clinical photoacoustic imaging of cancer," Ultrasonography, vol. 35, pp. 267-280, 2016.

[11] S. Zackrisson, S. M. W. Y. van de Ven, and S. S. Gambhir, "Light In and Sound Out: Emerging Translational Strategies for Photoacoustic Imaging," Cancer Res., vol. 74, no. 4, pp. 979-1004, 2014.

[12] X. Wang, J. B. Fowlkes, J. M. Cannata, H. Changhong, and P. L. Carson, "Photoacoustic Imaging with A Commercial Ultrasound System and A Custom Probe," Ultrasound Med. Biol., vol. 37, no. 3, pp. 484-492, 2011.

[13] L. V. Wang and L. Gao, "Photoacoustic microscopy and computed tomography: from bench to bedside," Annu Rev Biomed Eng, vol. 16, pp. 155-185, 2014.

[14] S. Mallidi, G. P. Luke, and S. Emelianov, "Photoacoustic imaging in cancer detection, diagnosis, and treatment guidance," Trends Biotechnol., vol. 29, no. 5, pp. 213-221, 2011.

[15] L. V Wang and J. Yao, "A Practical Guide to Photoacoustic Tomography in the Life Sciences," Nat Methods, vol. 13, no. 8, pp. 627-638, 2016.

[16] L. V. Wang, "Multiscale photoacoustic microscopy and computed tomography," Nat. Photonics, vol. 3, pp. 503-509, 2009.

[17] C. Li, C. Kim, and L.V.Wang, "Photoacoustic Tomography and Ultrasound-Modulated Optical Tomography," in Handbook of Biomedical Optics, D. A. Boas, C. Pitris, and N. Ramanujam, Eds. New York, US: CRC Press, 2011, pp. 419-442.

[18] M. Mehrmohammadi, S. J. Yoon, D. Yeager, and S. Y. Emelianov, "Photoacoustic Imaging for Cancer Detection and Staging," Curr Mol Imaging, vol. 2, no. 1, pp. 89-105, 2013.

[19] K. Maslov and L. V Wang, "Photoacoustic imaging of biological tissue with intensity-modulated continuous-wave laser," J. Biomed. Opt., vol. 13, no. 2, pp. 1-5, 2008.

[20] J. Yao and L. V Wang, "Sensitivity of photoacoustic microscopy," Photoacoustics, vol. 2, pp. 87-101, 2014.

[21] J. Xia, J. Yao, and L. V Wang, "Photoacoustic Tomography: Principles and Advances," Prog. Electromagn. Res., vol. 147, pp. 122, 2014.

[22] D. V Bageshwar, A. S. Pawar, V. V Khanvilkar, and V. J. Kadam, "Photoacoustic Spectroscopy and Its Applications - A Tutorial Review," Eurasian J. Anal. Chem., vol. 5, no. 2, pp. 187-203, 2010.

[23] T. J. Allen and P. C. Beard, "High power visible light emitting diodes as pulsed excitation sources for biomedical photoacoustics," Biomed. Opt. Express, vol. 7, no. 4, pp. 1260-1270, 2016.

[24] T. Wang, S. Nandy, H. S. Salehi, P. D. Kumavor, and Q. Zhu, "A low-cost photoacoustic microscopy system with a laser diode excitation," Biomed. Opt. Express, vol. 5, no. 9, pp. 20097-20102, 2014.

[25] B. Soroushian and X. Yang, "Measuring non-radiative relaxation time of fluorophores with biomedical applications by photoacoustic effect," Biomed. Opt. Express, vol. 2, no. 10, pp. 2749-2760, 2011.

[26] R. G. M. Kolkman, W. Steenbergen, and T. G. van Leeuwen, "In vivo photoacoustic imaging of blood vessels with a pulsed laser diode," Lasers Med Sci, vol. 21, pp. 134-139, 2006.

[27] P. Beard, "Biomedical photoacoustic imaging," Interface Focus, vol. 1, pp. 602-631, 2011.

[28] R. A. Convissar, Principles and Practice of Laser Dentistry. St. Louis, Missouri: Mosby Elsevier, 2011.

[29] P. Mohajerani, S. Kellnberger, and V. Ntziachristos, "Frequency domain optoacoustic tomography using amplitude and phase," Photoacoustics, vol. 2, no. 3, pp. 111-118, 2014. 